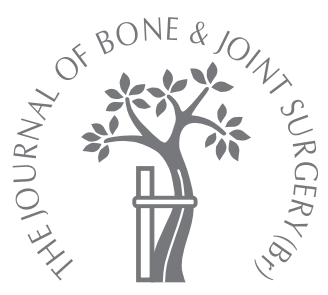

T. F. Oswald, F. K. Gould

From the Freeman Hospital, Newcastle upon Tyne, England

I. T. F. Oswald, MRCP, FRCPath, Specialist Registrar in Medical Microbiology

Royal Victoria Infirmary, Queen Victoria Road, Newcastle upon Tyne, NE1 4LP, UK.

E. K. Gould, MRCPath, FRCPath, Consultant Microbiologist

Freeman Hospital, High Heaton, Newcastle upon Tyne, NE7 7DN, UK.

Correspondence should be sent to $\mathrm{Dr}$ T. F. Oswald; email: t.oswald@doctors.org.uk

(C)2008 British Editorial Society of Bone and Joint Surgery doi:10.1302/0301-620X.90B7. $21110 \$ 2.00$

$J$ Bone Joint Surg $[\mathrm{Br}]$ 2008:90-B:825-6.

VOL. 90-B, No. 7, JULY 2008
EDITORIAL

\section{Dental treatment and prosthetic joints}

\author{
ANTIBIOTICS ARE NOT THE ANSWER!
}

\author{
The routine administration of prophylactic antibiotics for dental interventions to prevent \\ haematogenous spread of infections to prosthetic joints is a contentious issue. In this \\ editorial we discuss the potentially harmful effects of this practice and propose an \\ alternative solution.
}

We wish to commend the authors of the very thorough annotation, in the current issue of the Journal, of antibiotic prophylaxis before dental procedures in patients with hip and knee replacements; ${ }^{1}$ we agree almost entirely with their conclusions and recommendations. However, there are some points which merit further discussion and some additional matters which we wish to address.

When the first prosthetic hip was implanted in 1938, antibiotics were in the early stages of development. ${ }^{2}$ They appeared to be the miracle weapon in the fight against infection and revolutionised treatment. In orthopaedic surgery the introduction of antibiotic prophylaxis reduced the rate of postoperative infection from between $15 \%$ and $25 \%$, to $1 \%{ }^{3}$ However, although antibiotics have been increasingly used and misused, they have exerted a powerful influence on bacteria and have given rise to the development of resistance. This has become a global problem and has resulted in considerable morbidity, mortality and cost. The most effective way of controlling resistance is by reducing the use of antibiotics and thereby decreasing the selection process. This can be achieved by education and the implementation of strict policies in the use of antibiotics. Consequently, it is imperative that the evidence is reviewed rigorously when the benefit from the use of antibiotics is unclear.

The publication of the annotation ${ }^{1}$ is timely since the National Institute for Clinical Excellence (NICE) ${ }^{4}$ has recently given recommendations for prophylaxis against infective endocarditis. They concluded that antibiotics should not be recommended as prophylaxis for the prevention of infective endocarditis during invasive dental procedures. This is in agreement with the 2004 Cochrane review by Oliver, Roberts and Hooper. ${ }^{5}$

There is a lack of robust evidence linking dental procedures to an increased risk of infection of prosthetic joints. Routine daily activities such as tooth-brushing and chewing produce an equivalent or even greater bacteraemia than from a single dental treatment. Consequently, the risk of cumulative bacteraemia over time due to these daily activities will be far greater than from a dental procedure, particularly when associated with poor dentition. ${ }^{6,7}$

The overall risk of infection in hip replacement is less than $1 \%$ and in knee replacements less than $2 \%$, with late infection due to haematogenous spread being particularly rare. ${ }^{8}$ Only a very small number of these infections are caused by oral streptococci. These organisms can also arise from other sources such as the colon. A review of the literature has shown that only a few cases of infection have been attributed to recent dental procedures, mostly in the presence of a concomitant dental infection. ${ }^{1,3}$ It seems reasonable to conclude that spontaneous bacteraemia resulting from routine daily activities, particularly in the presence of poor dentition, is more likely to have been the cause in most cases. Since the degree of the bacteraemia is reduced by good oral hygiene, best practice should incorporate screening by a dentist to eliminate current dental disease before the replacement procedure, with advice on the maintenance of good oral hygiene following surgery.

Antibiotic prophylaxis has not been shown to effectively prevent dental-associated infections. The incidence and level of bacteraemia are merely reduced and infections still occur 
after appropriate prophylaxis. The number of patients who would have to be treated to prevent the occurrence of such an unlikely infection is, therefore, extremely high.

The authors of the annotation 1 postulate that the use of topical antibiotics or oral antiseptic agents could replace that of systemic antibiotics as prophylaxis. We consider that since there is insufficient evidence to support the link between dentistry and prosthetic joint infection, and since the routine administration of antibiotic prophylaxis is likely to be ineffective, antibiotic prophylaxis should not be given routinely.

Regardless of the risks of creating antibiotic resistance, the possible adverse effects on the individual patient must also be considered. Non-fatal side-effects such as gastrointestinal upsets and rash are relatively common $(1 \%$ to $10 \%$ ) and, although unpleasant, are self-limiting. ${ }^{5}$ Anaphylaxis, although rare, is associated with a significant rate of mortality of approximately $10 \%$, and would result in more deaths than might occur as a consequence of infected replacements. ${ }^{5}$ In addition, the risk of developing infection from Clostridium difficile must not be treated lightly, since it is also associated with significant morbidity and mortality. This is particularly pertinent to those aged over 65 years, who are at the greatest risk of such an infection.

Finally, cost implications must be considered. Several studies have shown that the overall cost of administering routine antibiotic prophylaxis is greater than that when prophylaxis is not given. ${ }^{1,3}$ This is partly due to the cost of managing adverse drug reactions in the patients receiving antibiotics.
In summary, there is no evidence to link prosthetic joint infections to dental procedures and none to prove that antibiotic prophylaxis is effective. The continued use of antibiotics would be expensive, contribute to an increase in bacterial resistance, lead to increased morbidity as a result of adverse side-effects and antibiotic-associated infections and increase the risk of death. It is clear that better oral hygiene is the answer rather than the administration of antibiotics. It would be more logical to use the funding which would be required for routine antibiotic prophylaxis, to provide high-quality dental care.

No benefits in any form have been received or will be received from a commercial party related directly or indirectly to the subject of this article.

\section{References}

1. Uçkay I, Pittet D, Bernard L et al. Antibiotic prophylaxis before invasive dental procedures in patients with arthroplasties of the hip and knee. J Bone Joint Surg [Br] 2008;90-B:833-8.

2. Wiles P. The surgery of the osteoarthritic hip. Br J Surg 1958;45:488-97.

3. Seymour RA, Whitworth JM, Martin M. Antibiotic prophylaxis for patients with joint prostheses: still a dilemma for dental practitioners. Br Dent J 2003;194:649-53.

4. No authors listed. NICE Short Clinical Guidelines Technical Team. Prophylaxis against infective endocarditis. Antimicrobial prophylaxis against infective endocarditis. London: National Institute for Health and Clinical Excellence. 2008. http:// www.nice.org.uk/CG064 (date last accessed 31 March 2008).

5. Oliver R, Roberts GJ, Hooper L. Penicillins for the prophylaxis of bacteria endocarditis in dentistry. Cochrane Database Syst Rev 2004; CD003813. http:// mrw.interscience.wiley.com/cochrane/clsysrev/articles/CD003813/frame.html (date last accessed 31 March 2008).

6. Gould FK, Elliott TS, Foweraker J, et al. Guidelines for the prevention of endocarditis: report of the Working Party of the British Society of Antimicrobial Chemotherapy. J Antimicrob Chemother 2006;57:1035-42.

7. Wilson W, Taulbert KA, Gewitz M, et al. Prevention of infective carditis: guidelines from the American Heart Association. Circulation 2007;116:1736-54.

8. Zimmerli W, Trampuz A, Ochsner PE. Prosthetic-joint infections. N Engl J Med 2004:351:1646-54 